Manchester Institute for Mathematical Sciences

School of Mathematics

The University of Manchester

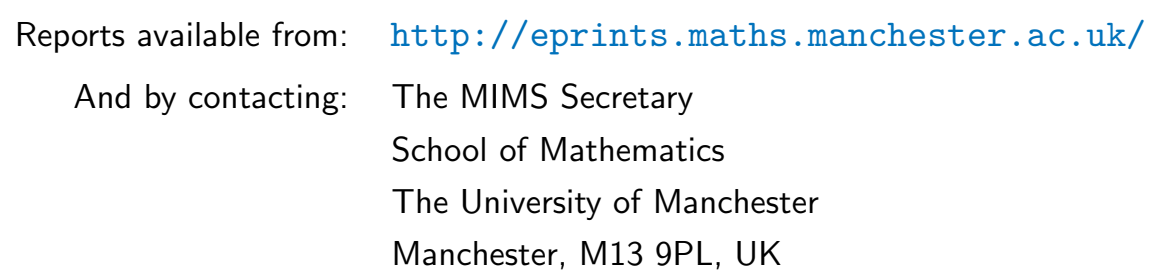

ISSN 1749-9097 


\title{
A SUFFICIENT CONDITION FOR THE EXISTENCE OF HAMILTONIAN BIFURCATIONS WITH CONTINUOUS ISOTROPY
}

\author{
JAMES MONTALDI AND MIGUEL RODRÍGUEZ-OLMOS
}

\begin{abstract}
We present a framework for the study of the local qualitative dynamics of equivariant Hamiltonian flows specially designed for points in phase space with nontrivial isotropy. This is based on the classical construction of structure-preserving tubular neighborhoods for Hamiltonian Lie group actions on symplectic manifolds. This framework is applied to obtaining concrete and testable conditions guaranteeing the existence of bifurcations from symmetric branches of Hamiltonian relative equilibria.
\end{abstract}

\section{INTRODUCTION}

In this short note we will outline a method for studying the qualitative dynamics of symmetric Hamiltonian systems near relative equilibria, with emphasis in those which lie in singular leaves of the momentum map, or equivalently, have continuous isotropy. Using this method we will state a sufficient, easily testable condition for the existence of a bifurcation from a continuous family of relative equilibria parametrized by momentum. For reasons of space this paper is of an expository nature and an announcement of some of the results contained in [5] where a more detailed analysis is carried out.

Recall that a $G$-Hamiltonian system is a quintuple $(\mathcal{P}, \omega, G, \mathbf{J}, h)$, where $(\mathcal{P}, \omega)$ is a smooth symplectic manifold, $G$ is a Lie group acting on $\mathcal{P}$ by symplectomorphisms, $h \in C^{\infty}(\mathcal{P})$ is a $G$-invariant Hamiltonian function and $\mathbf{J}: \mathcal{P} \rightarrow \mathfrak{g}^{*}$ is a $\mathrm{Ad}^{*}$-equivariant momentum map satisfying

$$
\iota_{\xi_{\mathcal{P}}} \omega=\mathbf{d}\langle\mathbf{J}(\cdot), \xi\rangle, \quad \forall \xi \in \mathfrak{g} .
$$

Here $\mathfrak{g}$ denotes the Lie algebra of $G$ and $\xi_{\mathcal{P}}(z)=\left.\frac{d}{d t}\right|_{t=0} \exp t \xi \cdot(z)$ is the evaluation at $z$ of the fundamental vector field associated to the Lie algebra element $\xi \in \mathfrak{g}$. Throughout this paper we will assume that $G$ is compact.

The associated dynamical system on $\mathcal{P}$ is the flow of the Hamiltonian vector field defined implicitly by

$$
\iota_{X_{h}} \omega=\mathbf{d} h .
$$

This flow is $G$-invariant, thus sending group orbits to group orbits for all times. A well known theorem by Noether states that $\mathbf{J}$ is constant along integral curves of $X_{h}$.

School of Mathematics. University of Manchester. Manchester M13 9PL, UK j.montaldi@manchester.ac.uk.

Department of Applied Mathematics IV. Technical University of Catalonia. Barcelona, Spain miguel.rodriguez.olmos@upc.edu. 
A relative equilibrium is an integral curve of the dynamical system that is contained in a group orbit. They can be characterized (see [3]) as those points $z \in \mathcal{P}$ for which there exists an element $\xi \in \mathfrak{g}$ such that

$$
X_{h}(z)=\xi_{\mathcal{P}}(z) .
$$

The Lie algebra element $\xi$, which depends on $z$, is called a velocity for the relative equilibrium $z$. Notice that it follows from the definition that if $z$ is a relative equilibrium with velocity $\xi$, then every point in the integral curve of $X_{h}$ through $z$ is also a relative equilibrium admitting $\xi$ as a velocity. Moreover, it can be proved that $\xi$ is an element of $\mathfrak{g}_{\mu}$, the Lie algebra of the stabilizer of $\mu=\mathbf{J}(z)$ under the coadjoint representation of $G$.

From (11) we obtain a key observation that will be crucial for this paper. Suppose that the stabilizer of $z$, denoted $G_{z}$, has a non-trivial Lie algebra $\mathfrak{g}_{z}$ (which is equivalent to $G_{z}$ not being discrete); if $z$ is a relative equilibrium with velocity $\xi$, then for any $\gamma \in \mathfrak{g}_{z}$ the element $\xi+\gamma$ is also a velocity for the same relative equilibrium. This non-uniqueness in the assignment of a velocity to a relative equilibrium will have important consequences for the local dynamics of $G$-Hamiltonian systems near relative equilibria. Another standard fact is that if $z$ is a relative equilibrium with velocity $\xi$ then for any group element $g$, the point $g \cdot z$ is a relative equilibrium with velocity $\operatorname{Ad}_{g} \xi$. We will consider all relative equilibria related in this way as equivalent.

Another important characterization of Hamiltonian relative equilibria is the following (see [3]). A point $z \in \mathcal{P}$ is a relative equilibrium with velocity $\xi$ if and only if

$$
\mathbf{d} h_{\xi}(z)=0,
$$

where the function $h_{\xi}$ is the augmented Hamiltonian, defined by

$$
h_{\xi}(z)=h(z)-\langle\mathbf{J}(z), \xi\rangle .
$$

By the previous considerations we have that if $z$ is a relative equilibrium with velocity $\xi$ then also $\mathbf{d} h_{\xi+\gamma}(z)=0$ for every element $\gamma \in \mathfrak{g}_{z}$.

The organization of the article is as follows: Section 2 contains some generalities about Hamiltonian actions and Hamiltonian relative equilibria, including a brief review of the construction of the Marle-Guillemin-Sternberg form (MGS). Then we introduce the so-called bundle equations as introduced in [8] and [9], which can be thought as Hamilton's equation for an invariant Hamiltonian function on the tubular model provided by the MGS form. Next, we show the main novelty of our approach which consists in including the explicit role of the isotropy of the group action into the bundle equations, obtaining our main tool, the system of equations (11) (12) and (13). Section 3 contains the main result of this note, Theorem 3.1 which gives a test for the existence of bifurcations from a branch of relative equilibria parametrized by momentum values. This test is illustrated with an example in Section 4.

A criterion for determining the stability of these relative equilibria with continuous isotropy was developed in a recent paper by the authors [4].

\section{The Bundle Equations around Relative Equilibria}

We will assume from now on that $z \in \mathcal{P}$ is a relative equilibrium with velocity $\xi$ and momentum $\mu=\mathbf{J}(z)$. We are interested in studying the local dynamics 
around $z$ and in obtaining conditions characterizing nearby relative equilibria. For that we will use the usual approach which consists in replacing $\mathcal{P}$ by the MGS normal form at $z$. This provides a tubular neighborhood of the group orbit $G \cdot z$ adapted to the existing geometry and symmetries ([2, 1]). We will now briefly recall its construction. Note that by the equivariance of $\mathbf{J}$ we have the inclusions $\mathfrak{g}_{z} \subset \mathfrak{g}_{\mu} \subset \mathfrak{g}$. Therefore, by the compactness hypothesis on $G$ we can define $G_{z^{-}}$ invariant subspaces $\mathfrak{m} \subset \mathfrak{g}_{\mu}, \mathfrak{q} \subset \mathfrak{g}$ and $N \subset \operatorname{ker} T_{z} \mathbf{J}$ satisfying

$$
\mathfrak{g}_{\mu}=\mathfrak{g}_{z} \oplus \mathfrak{m}, \quad \mathfrak{g}=\mathfrak{g}_{z} \oplus \mathfrak{m} \oplus \mathfrak{q} \quad \text { and } \quad \operatorname{ker} T_{z} \mathbf{J}=T_{z}\left(G_{\mu} \cdot z\right) \oplus N .
$$

These splittings are chosen to be $G_{z}$-invariant and have associated dual ones. In particular we will use the induced splitting of $\mathfrak{g}_{\mu}^{*}$

$$
\mathfrak{g}_{\mu}^{*}=\mathfrak{g}_{z}^{*} \oplus \mathfrak{m}^{*} .
$$

It is a standard fact that $(N, \Omega)$, where $\Omega$ is the restriction to $N$ of $\omega(z)$, is a symplectic vector space and that the linear action of $G_{z}$ on $N$ is Hamiltonian, with associated equivariant momentum map $\mathbf{J}_{N}: N \rightarrow \mathfrak{g}_{z}^{*}$ defined by

$$
\left\langle\mathbf{J}_{N}(v), \eta\right\rangle=\frac{1}{2} \Omega(\eta \cdot v, v), \quad \forall \eta \in \mathfrak{g}_{z}, v \in N .
$$

The linear space $N$ is called the symplectic normal space at $z$ for the $G$-action on $\mathcal{P}$.

Let $Y:=G \times{ }_{G_{z}}\left(\mathfrak{m}^{*} \times N\right)$ be the quotient space for the action of $G_{z}$ on $G \times \mathfrak{m}^{*} \times N$ given by

$$
h \cdot(g, \rho, v)=\left(g h^{-1}, \operatorname{Ad}_{h^{-1}}^{*} \rho, h \cdot v\right) .
$$

This quotient happens to be equipped with a symplectic 2-form $\omega_{Y}$ in a neighborhood of the zero section $G \times \times_{G_{z}}(\{0\} \times\{0\})$. Moreover, the Lie group $G$ has a natural Hamiltonian action on $Y$ given by

$$
g^{\prime} \cdot[g, \rho, v]=\left[g^{\prime} g, \rho, v\right],
$$

which admits the momentum map $\mathbf{J}_{Y}: Y \rightarrow \mathfrak{g}^{*}$ given by

$$
\mathbf{J}_{Y}([g, \rho, v])=\operatorname{Ad}_{g^{-1}}^{*}\left(\mu+\rho+\mathbf{J}_{N}(v)\right) .
$$

Notice also that from (7) and (6) we have that the stabilizer of a point in $Y$ is

$$
G_{[g, \rho, v]}=g\left(\left(G_{z}\right)_{\rho} \cap\left(G_{z}\right)_{v}\right) g^{-1},
$$

where the right hand term is the stabilizer of $v$ under the linear representation of $G_{z}$ on $N$.

The Hamiltonian Tube Theorem guarantees the existence of a map $\varphi: Y=$ $G \times{ }_{G_{z}}\left(\mathfrak{m}^{*} \times N\right) \rightarrow \mathcal{P}$ which sends $[e, 0,0]$ to $z$, and which is a symplectomorphism between a $G$-invariant neighborhood of the zero section of $Y$ and a $G$-invariant neighborhood of the group orbit $G \cdot z$, and intertwines the Hamiltonian actions of $G$ on the domain and the target, thereby satisfying $\mathbf{J}_{Y}=\mathbf{J} \circ \varphi$ wherever this expression is defined.

The idea exploited in [ 8] and [9] consists in taking the pullback by $\varphi$ of the Hamiltonian vector field $X_{h}$ to $Y$ and then lift it to $G \times \mathfrak{m}^{*} \times N$. In these two references the differential equations for the flow of a particular choice of the lifted vector field are obtained, providing a general framework to study the local dynamics of $X_{h}$ near $G \cdot z$. These are called the bundle equations, and we now reproduce them. 
Note that $h \circ \varphi$ is a $G$-invariant function on $Y$, so it can be identified with a $G_{z^{-}}$ invariant function $\bar{h}$ on $\mathfrak{m}^{*} \times N$. Notice also that a vector field $X \in \mathfrak{X}\left(G \times \mathfrak{m}^{*} \times N\right)$ which is the lift of a vector field on $Y$ can be expressed as

$$
X(g, \rho, v)=\left(g \cdot\left(X_{\mathfrak{g}}(g, \rho, v)\right), X_{\mathfrak{m}^{*}}(g, \rho, v), X_{N}(g, \rho, v)\right),
$$

where $X_{\mathfrak{g}}(g, \rho, v) \in \mathfrak{g}, X_{\mathfrak{m}} *(g, \rho, v) \in \mathfrak{m}^{*}$, and $X_{N}(g, \rho, v) \in N$. The bundle equations on $G \times \mathfrak{m}^{*} \times N$ are then given by:

$$
\begin{aligned}
X_{\mathfrak{g}}(g, \rho, v) & =D_{\mathfrak{m}^{*}} \bar{h}(\rho, v) \\
X_{\mathfrak{m}^{*}}(g, \rho, v) & =\mathbb{P}_{\mathfrak{m}^{*}}\left(\operatorname{ad}_{D_{\mathfrak{m}^{*}} \bar{h}(\rho, v)}^{*}\left(\rho+\mathbf{J}_{N}(v)\right)\right) \\
X_{N}(g, \rho, v) & =\Omega^{\sharp}\left(D_{N} \bar{h}(\rho, v)\right) .
\end{aligned}
$$

Where $D_{\mathfrak{m}^{*}} \bar{h}$ and $D_{N} \bar{h}$ denote the partial derivatives of $h \in C^{G_{z}}\left(\mathfrak{m}^{*} \times N\right)$ with respect to $\mathfrak{m}^{*}$ and $N$ respectively. Here the symbol $\mathbb{P}$ denotes the canonical projection onto the subspace indicated by the subscript relative to a splitting of some vector space, which will be clear from the context. As shown in [8, 9], these are the differential equations for the flow of the unique local vector field $X$ on $G \times \mathfrak{m}^{*} \times N$ which is a lift of $\varphi^{*} X_{h} \in \mathfrak{X}(Y)$ and satisfies the condition

$$
\mathbb{P}_{\mathfrak{g}_{z}}\left(X_{\mathfrak{g}}\right)=0 .
$$

$\varphi^{*} X_{h}=X_{h \circ \varphi}$, where $X_{h \circ \varphi}$ is the solution of Hamilton's equations

The main novelty of our approach consists in studying simultaneously all the lifts of $\varphi^{*} X_{h}$ and not only the one prescribed by condition (10). For that, it is clear that one has to add the set of vector fields on $G \times \mathfrak{m}^{*} \times N$ that are tangent to the $G_{z}$-orbits of the action (6). These vertical fields are generated by the set of fields of the form

$$
X(g, \rho, v)=\left(g \cdot \gamma, \operatorname{ad}_{\gamma}^{*} \rho,-\gamma \cdot v\right),
$$

for $\gamma \in \mathfrak{g}_{z}$. In [5] we show that the flow of the most general lift of $\varphi^{*} X_{h}$ is described by the modified bundle equations

$$
\begin{aligned}
\dot{g} & =g \cdot\left(D_{\mathfrak{m}^{*}} \bar{h}(\rho, v)+\gamma\right) \\
\dot{\rho} & =\mathbb{P}_{\mathfrak{m}^{*}}\left(\operatorname{ad}_{D_{\mathfrak{m}} * \bar{h}(\rho, v)+\gamma}^{*}\left(\rho+\mathbf{J}_{N}(v)\right)\right) \\
\dot{v} & =\Omega^{\sharp}\left(D_{N} \bar{h}_{\gamma}(\rho, v)\right) .
\end{aligned}
$$

for arbitrary $\gamma \in \mathfrak{g}_{z}$ and

$$
\bar{h}_{\gamma}(\rho, v)=\bar{h}(\rho, v)-\left\langle\mathbf{J}_{N}(v), \gamma\right\rangle .
$$

\section{A Sufficient Condition for a symmetric Hamiltonian Bifurcation}

According to (11),(12) and (13), and using the non-degeneracy of the symplectic form $\Omega$ we can see that a point $[g, \rho, v] \in Y$, or equivalently, the point $\varphi([g, \rho, v]) \in \mathcal{P}$ near $z$ is a relative equilibrium for the vector field $\varphi^{*} X_{h}$ if, choosing $\gamma$ appropriately in order to take into account the directions tangent to the vertical vector fields, we have $\dot{\rho}=0$ and $\dot{v}=0$. That is

$$
\begin{aligned}
\mathbb{P}_{\mathfrak{m}^{*}}\left(\operatorname{ad}_{D_{\mathfrak{m} *} * \bar{h}(\rho, v)+\gamma}^{*}\left(\rho+\mathbf{J}_{N}(v)\right)\right) & =0 \\
D_{N} \bar{h}_{\gamma}(\rho, v) & =0 .
\end{aligned}
$$


In that case it follows easily from (11) that a velocity of the relative equilibrium is given by $\left.\xi=\operatorname{Ad}_{g^{-1}}\left(D_{\mathfrak{m}^{*}} \bar{h}(\rho, v)+\gamma\right)\right)$.

Notice that according to the splittings (3) and (4), $D_{\mathfrak{m}} * \bar{h}(\rho, v)+\gamma \in \mathfrak{g}_{\mu}$ and $\rho+$ $\mathbf{J}_{N}(v) \in \mathfrak{g}_{\mu}^{*}$. Therefore, if we assume that $G_{\mu}$ is a torus, then (14) is automatically satisfied, reducing the problem of characterizing relative equilibria to finding critical points on $N$ of the family of functions $\bar{h}_{\gamma}(\rho, \cdot)$ parametrized by $(\gamma, \rho) \in \mathfrak{g}_{z} \times \mathfrak{m}^{*}$. The following result is an application of this framework towards providing sufficient conditions for the existence of Hamiltonian bifurcations from a parametrized family of relative equilibria in a $G$-Hamiltonian system. Before we will fix some notation. We say that an injective map $\phi: V \rightarrow \mathcal{P}$, where $V$ is a (open neighborhood of 0 in a) vector space is a parametrized branch of relative equilibria if for every $w \in V$, the point $\phi(w)$ is a relative equilibrium, and all the relative equilibria of the branch are non-equivalent in the sense mentioned in the Introduction. That is, all the points of the branch belong to different $G$-orbits in $\mathcal{P}$. The relative equilibria of a branch are said to be of the same type if for every $w \in V$, there is some $g \in G$ such that $G_{\mathbf{J}(\phi(w))}=g G_{\mu} g^{-1}$ and $G_{\phi(w)}=g H g^{-1}$ for fixed subgroups $G_{\mu}, H \subset G$.

Theorem 3.1. Let $z \in \mathcal{P}$ be a relative equilibrium with momentum $\mathbf{J}(z)=\mu$ belonging to a parametrized branch $z: \mathfrak{m}^{*} \rightarrow \mathcal{P}$ of relative equilibria of the same type such that $z(0)=z$. Let $\xi(\rho) \in \mathfrak{g}$ be a continuous choice of velocities for $z(\rho)$ with $\xi(0)=\xi$ and suppose that $G_{\mu}$ is a torus. Let also $N_{\rho}$ be a continuous choice of symplectic normal spaces for $z(\rho)$ with $N_{0}=N$. They are all isomorphic as symplectic representations of $G_{z}$ by construction. Suppose also that there exists some $\gamma \in \mathfrak{g}_{z}$ and a subgroup $L \subset G_{z}$ satisfying

(i) For all $\rho$ in a neighborhood of $0,\left.\mathbf{d}_{z(\rho)}^{2} h_{\xi(\rho)+\gamma}\right|_{N_{\rho}^{L}}$ has an isolated eigenvalue $\sigma(\rho)$ (possibly multiple) that crosses 0 at $\rho=0$.

(ii) $\left.\operatorname{ker} \mathbf{d}_{z}^{2} h_{\xi+\gamma}\right|_{N^{L}}$ is at most two dimensional and $G_{z}$ contains a subgroup isomorphic to $\mathrm{SO}(n)$ that acts effectively on this space.

Then, for every $\left.v \in \operatorname{ker} \mathbf{d}_{z}^{2} h_{\xi+\eta}\right|_{N^{L}} \backslash\{0\}$ close enough to the origin there is a relative equilibrium $z^{\prime}(v)$ near $z$ with stabilizer containing $L$ and not contained in the branch $z(\rho)$.

Proof. First of all, using the independence of the $\mathfrak{g}_{z}$-component of the velocity of a relative equilibrium, we can choose $\xi(\rho) \in \mathfrak{m}^{*}$ according to the splitting (3).

Let $Y=G \times_{G_{z}}\left(\mathfrak{m}^{*} \times N\right)$ be the MGS model at the relative equilibrium $z$. Since the branch of relative equilibria is parametrized by $\mathfrak{m}^{*}$ it corresponds to the branch $[g(\rho), \rho, v(\rho)] \in Y$. After a reorganization of the points in this branch using the freedom in the group action this can be presented as $[e, \rho, v(\rho)]$. And, since $G_{\mu}$ is Abelian and all the points in this branch are relative equilibria of the same type, we have that $\left(G_{z}\right)_{v(\rho)}=G_{z}$ for all $\rho \in \mathfrak{m}^{*}$ (using (9)). Moreover, using the $G_{z}$-equivariant change of variables on $N$ given by $v \mapsto v-v(\rho)$ we induce the well defined $G$-equivariant symplectic diffeomorphism on $Y[g, \rho, v] \mapsto$ $[g, \rho, v-v(\rho)]$ and therefore we can assume that our original branch of relative equilibria on $\mathcal{P}$ corresponds on $Y$ to the family $[e, \rho, 0]$, with $\rho \in \mathfrak{m}^{*}$. And they all satisfy $D_{N} \bar{h}_{\gamma}(\rho, 0)=0$ for every $\rho \in \mathfrak{m}^{*}$ and $\gamma \in \mathfrak{g}_{z}$ (using (15) and (5)).

We will now need the following technical result in order to prove our bifurcation result. This is a straightforward application of the normal form of the momentum map in the MGS model of a G-Hamiltonian system, and the proof can be found in 5. 
Lemma 3.1. Under the previous assumptions, for every subgroup $L \subset G_{z}$ the $\mathfrak{m}^{*}$-parametrized families of bilinear forms $\left.D^{2}{ }_{N} \bar{h}_{\gamma}(\rho, 0)\right|_{N^{L}}$ and $\left.\mathbf{d}_{\varphi([e, \rho, 0])}^{2} h_{\xi(\rho)+\gamma}\right|_{N_{\rho}^{L}}$ have the same signature.

Now, using this lemma we can reduce the problem of finding bifurcating relative equilibria to finding elements $0 \neq v \in N$ such that there exists a $\gamma \in \mathfrak{g}_{z}$ for which

$$
D_{N} \bar{h}_{\gamma}(\rho, v)=0
$$

under the assumptions

A1. $D_{N} \bar{h}_{\gamma}(\rho, 0)=0$ for all $\rho \in \mathfrak{m}^{*}$, and

A2. there is an eigenvalue of the family of Hessians $\left.D_{N}^{2} \bar{h}_{\gamma}(\rho, 0)\right|_{N^{L}}$ that crosses 0 at $\rho=0$.

Let us call $f(\rho, \cdot) \in C^{\infty}(N)$ the $\mathfrak{m}^{*}$-parametrized function on $N$ defined by $f(\rho, v)=$ $\bar{h}_{\gamma}(\rho, v)$, and by $f^{L}$ its restriction to $N^{L}$. We will restrict our attention to finding critical points of $f(\rho, \cdot)$ in $N^{L}$ other than the origin which according to the Principle of Symmetric Criticality (see [6]) are exactly the critical points of $f^{L}(\rho, \cdot)$ since $f(\rho, \cdot)$ is $G_{z}$-invariant, and in particular $L$-invariant for any $\rho \in \mathfrak{m}^{*}$. Note that those are precisely the points satisfying $\left.D_{N} \bar{h}_{\gamma}(\rho, v)\right|_{N^{L}}=0$ with $0 \neq v \in N^{L}$. Also, recall that using the pullback property of Hessians, $D_{N^{L}}^{2} f^{L}(\rho, 0)=\left.D_{N}^{2} f(\rho, 0)\right|_{N^{L}}$. With these considerations we have that conditions A1 and A2 are equivalent to

A3. $D_{N^{L}} f^{L}(\rho, 0)=0$ for all $\rho \in \mathfrak{m}^{*}$, and

A4. There is an eigenvalue of the family of Hessians $D_{N^{L}}^{2} f^{L}(\rho, 0)$ that crosses 0 at $\rho=0$.

We will now assume that $\sigma(\rho)$, the eigenvalue that changes sign, has a one dimensional eigenspace for any value of the parameter. In particular this implies that $K=\operatorname{ker} D_{N^{L}}^{2} f(0,0)$ has dimension one. Using the Splitting Lemma [7] we can find a splitting $N^{L}=K \oplus S$ such that

$$
f^{L}(\rho, v)=g_{\rho}(x)+Q_{\rho}(s),
$$

where $Q_{\rho}$ is a family of quadratic forms, $y \in K, s \in S$ and $v=y+s$. Moreover, this can be chosen such that $K$ is the eigenspace corresponding to $\sigma(\rho)$, for every $\rho$.

Also, $g_{\rho}$ satisfies

$$
g_{\rho}^{\prime}(0)=0
$$

for every value of $\rho$. Since critical points of $f^{L}(\rho, \cdot)$ must be of the form $v=x+0$ with $x$ a critical point of $g_{\rho}$ we have reduced the problem to finding pairs $(\rho, x) \in$ $\mathfrak{m}^{*} \times K$ for which

$$
g_{\rho}^{\prime}(x)=0
$$

Using (17) and the condition on $\sigma(\rho)$ we can write

$$
g_{\rho}(x)=g_{\rho}(0)+\frac{1}{2} x^{2}\left(\sigma(\rho)+x l_{\rho}(x)\right),
$$

for some family of functions $l_{\rho}$. This implies that

$$
g_{\rho}^{\prime}(x)=x\left(\sigma(\rho)+x l_{\rho}(x)\right)+\frac{1}{2} x^{2}\left(l_{\rho}(x)+x l_{\rho}^{\prime}(x)\right)=x\left(\sigma(\rho)+x b_{\rho}(x)\right)=x j_{\rho}(x),
$$

with $j_{\rho}(x)=\sigma(\rho)+x b_{\rho}(x)$. Notice that by hypothesis, as a function of $\rho$, the function $j_{\rho}(0)$ changes sign precisely at $\rho=0$. Therefore, by continuity, for any 
$0 \neq \bar{x} \in K$ there must be at least one value $\bar{\rho}$ satisfying $j_{\bar{\rho}}(\bar{x})=0$. We have then that $D_{N} \bar{h}_{\gamma}(\bar{\rho}, \bar{x})=0$ and therefore, for any $g \in G$ and any $0 \neq \bar{x} \in K$ the point $\varphi([g, \bar{\rho}, \bar{x}+v(\bar{\rho})] \in \mathcal{P}$ is a relative equilibrium inequivalent to any of those contained in the original branch.

In the case that $\operatorname{dim} \operatorname{ker} D_{N^{L}}^{2} f(0,0)=n \geq 1$, if $G_{z}$ contains a subgroup isomorphic to $\mathrm{SO}(n)$ acting effectively on this space, then the functions $f^{L}(\rho, \cdot)$ on $N^{L}$ and $g_{\rho}$ on $K$ are $\mathrm{SO}(n)$ invariant. This means in particular that $g$ is a function of $\|x\|^{2}$ and we can reduce this to the one-dimensional problem.

Remark. Note that the possible bifurcations from a branch of relative equilibria is strongly influenced by the existence of a continuous isotropy group $G_{z}$. Had $G_{z}$ been discrete, $\xi(\rho)$ along the branch would have been completely determined, and the freedom in $\gamma \in \mathfrak{g}_{z}$ would not exist in order to fullfill condition (i) of Theorem 3.1. In fact, in a typical situation a relative equilibrium $z$ could be formally stable, which implies that the velocity $\xi$ can be chosen in such a way that $\left.\mathbf{d}_{z}^{2} h_{\xi}\right|_{N}$ is definite and still there could be a choice of $\gamma \in \mathfrak{g}_{z}$ such that condition (i) of Theorem 3.1 is satisfied. This is more likely to happen the larger is $G_{z}$, i.e. the more symmetric is the branch $G_{z}$.

\section{Example.}

Let $z$ be a relative equilibrium of a $G$-Hamiltonian system with $G_{\mu}=\mathbb{T}^{2}$ and $G_{z}=S^{1}$. If we identify $\mathfrak{g}_{\mu}$ with $\mathbb{R}^{2}$ then we can identify $\mathfrak{g}_{z}$ and $\mathfrak{m}^{*}$ with $\mathbb{R}$.

Suppose that the symplectic normal space at $z, N$, is isomorphic to $\mathbb{R}^{4}$, where in a Darboux basis the symplectic matrix $\Omega$ takes the form

$$
\Omega=\left(\begin{array}{llll}
0 & 1 & 0 & 0 \\
-1 & 0 & 0 & 0 \\
0 & 0 & 0 & 1 \\
0 & 0 & -1 & 0
\end{array}\right)
$$

Suppose that in the linear coordinates $\left\{x_{1}, y_{1}, x_{2}, y_{2}\right\}$ associated to this basis we have

$$
\bar{h}(\rho, v)=\frac{\rho+1}{2}\left(x_{1}^{2}+y_{1}^{2}\right)+\frac{1}{4}\left(x_{1}^{2}+y_{1}^{2}\right)^{2}-\frac{1}{2}\left(x_{2}^{2}-y_{2}^{2}\right),
$$

where $v=\left(x_{1}, y_{1}, x_{2}, y_{2}\right)$, and $\rho \in \mathfrak{m}^{*}$.

Suppose also that in this basis, the symplectic representation of $G_{z}=S^{1}$ on $N$ is given by

$$
S^{1} \ni \theta \mapsto\left(\begin{array}{ll}
R_{\theta} & 0 \\
0 & R_{\theta}
\end{array}\right),
$$

with $R_{\theta}$ being the standard rotation of angle $\theta$ on $\mathbb{R}^{2}$. The associated momentum map has the expression

$$
\mathbf{J}_{N}(v)=\frac{1}{2}\left(x_{1}^{2}+y_{1}^{2}+x_{2}^{2}+y_{2}^{2}\right) .
$$

Therefore we can obtain the expression for $\bar{h}_{\gamma}$ as

$$
\bar{h}_{\gamma}(\rho, v)=\frac{\rho+1-\gamma}{2}\left(x_{1}^{2}+y_{1}^{2}\right)+\frac{1}{4}\left(x_{1}^{2}+y_{1}^{2}\right)^{2}-\frac{1+\gamma}{2}\left(x_{2}^{2}-y_{2}^{2}\right)
$$


Since $G_{\mu}$ is Abelian, one can easily check using (15) that $[e, \rho, 0] \in Y$ is a branch of relative equilibria for any $\rho$ in some interval in $\mathfrak{m}^{*}$ containing the origin. Also, we can check that this is true for any choice of $\gamma$, which will be important later.

Using the freedoom in $\mathfrak{g}_{z}$, we fix $\gamma=1$, then we have that

$$
D_{N}^{2} \bar{h}_{\gamma}(\rho, 0)=\left(\begin{array}{llll}
\rho & 0 & 0 & 0 \\
0 & \rho & 0 & 0 \\
0 & 0 & -1 & 0 \\
0 & 0 & 0 & 1
\end{array}\right) .
$$

In order to apply Theorem 3.1 we choose $L=\{e\}$. Then we see that $\rho$ is the only eigenvalue that crosses zero. Then $\left.\operatorname{ker} D_{N}^{2} \bar{h}_{\gamma}(0,0)\right|_{N^{L}}=\left\{x_{1}, y_{1}\right\}$, which, from (19) supports an effective representation of $G_{z}=S^{1}$. Therefore, the result applies and there exists a branch of relative equilibria of the form $\left[e, \rho\left(x_{1}, x_{2}\right),\left(x_{1}, x_{2}, 0,0\right)\right]$, for every $\left(x_{1}, x_{2}\right) \neq 0$ close enough to the origin, which is not equivalent to the original branch. It is straightforward to obtain by a direct calculation that this bifurcating branch is characterized by $\rho\left(x_{1}, x_{2}\right)=x_{1}^{2}+x_{2}^{2}$.

Notice also that in this example, as remarked after Theorem 3.1 the existence of a bifurcating branch is a direct consequence of the existence of a continuous isotropy group $G_{z}$. Indeed, if $G_{z}$ was discrete, its Lie algebra would have been trivial and therefore one could not choose $\gamma$ other than zero. But in that case $D_{N}^{2} \bar{h}_{\gamma}(0,0)$ clearly is non-degenerate for any $\rho$ near zero. The implicit function theorem guarantees then that in the case of discrete $G_{z}$ the branch $[e, \rho, 0]$ is the only solution to (15) (apart from group translations), which means that no bifurcations can occur.

\section{Aknowledgments}

M. R.-O. wishes to thank the Hanoi National University of Education and the organizers of the workshop "Geometrical methods in Dynamics and Topology" for the kind invitation to attend this meeting. His research has been funded by the European Union through the Marie Curie Intra European Fellowship PIEFGA2008-220239, the Marie Curie Reintegration Grant PERG-GA-2010-27697 and by MEC (Spain) through the grant MTM2009-13383.

\section{REFERENCES}

[1] Guillemin, V. and Sternberg, S. [1984], A normal form for the moment map. In Differential Geometric Methods in Mathematical Physics, S. Sternberg ed. Mathematical Physics Studies, 6, D. Reidel.

[2] Marle, C.-M. [1985], Modèle d'action hamiltonienne d'un groupe de Lie sur une variété symplectique. Rend. Sem. Mat. Univ. Politec. Torino $432227-251$.

[3] Marsden, J.E. [1992], Lectures on Mechanics. Lecture Note Series 174, LMS, Cambridge University Press.

[4] Montaldi, J. and Rodríguez-Olmos, M. [2011], On the stability of Hamiltonian relative equilibria with continuous isotropy. Nonlinearity 242777.

[5] Montaldi, J. and Rodríguez-Olmos, M. Hamiltonian relative equilibria with continuous isotropy. In preparation.

[6] Palais, R.S. [1979] The principle of symmetric criticality. Comm. Math. Phys. 69, 1, 19-30.

[7] Poston T. and Stewart I. [1996], Catastrophe Theory and Applications. Dover.

[8] Roberts, M. and Sousa-Dias, M.E. [1997], Bifurcations from relative equilibria of Hamiltonian systems. Nonlinearity 10 1719-1738.

[9] Roberts, M., Wulff, C. and Lamb J.S. [2002], Hamiltonian systems near relative equilibria. J. Differential Equations 1792 562-604. 